\title{
V-type Three-Level Laser Coupled to Squeezed Vacuum Reservoir
}

\author{
Gomera Biyazn", Misrak Gatahun \\ Department of Physics, Hawassa University, Hawassa, Ethiopia \\ Email address: \\ biyazngomera17@gmail.com (G. Biyazn)
}

\section{To cite this article:}

Gomera Biyazn, Misrak Gatahun. V-type Three-Level Laser Coupled to Squeezed Vacuum Reservoir. American Journal of Optics and Photonics. Vol. 9, No. 1, 2021, pp. 1-9. doi: 10.11648/j.ajop.20210901.11

Received: February 1, 2021; Accepted: February 16, 2021; Published: March 3, 2021

\begin{abstract}
The squeezing, entanglement, and statistical properties of cavity modes produced by V-type three-level laser coupled to squeezed vacuum reservoir were studied employing the steady state solutions of the expectation values of the cavity mode operators. The study showed that a light in squeezing state can be produced by the system under consideration under the condition that the cavity decay constant is larger than linear gain coefficient and the squeezing occurs in the plus-quadrature. The cavity modes do not exhibit entanglement properties. It is also observed that the degree of squeezing increases with the linear gain coefficient and squeezing parameter. We also determined the mean and variance of the photon number, intensity difference fluctuations, and the second order correlation function of the two modes. The mean and variance of the photon number as well as the correlation function of two modes decrease as the difference of the probability of the two states at initial time increases. The photons have super-poissionian statistics. The point, $\eta$, at which the maximum intensity difference fluctuation occurred depends on linear gain coefficient but not squeezing parameter. The result shows that the correlation of the two modes decreases as $\eta$ the linear gain coefficient and squeezing parameter.
\end{abstract}

Keywords: Entanglement, EPR, Intensity Difference, Laser, Quadrature Squeezing

\section{Introduction}

The heart of quantum optics is the interaction of matter with radiation [1]. The light beam consists of photons that carry energies which contribute to the interaction between the light and the gravitational force [2].

Squeezing is one of the non-classical features of light in which the quantum noise in one quadrature is below quantum standard limit at which in the expense of enhanced fluctuation in the conjugate quadrature, with the product of the variance in the two quadrature satisfying the uncertainty relation [3-15]. Quantum squeezing and entanglement have both fundamental scientific significance and potential applications in quantum metrology and quantum information processing [7]. A three-level laser may be defined as a quantum optical system in which three-level atoms initially prepared in a coherent superposition of their two levels, are injected into a cavity coupled to a vacuum reservoir via a single-port mirror. Three-level system involves two dipole transients [8]. The V-type three-level laser in which the atoms' one ground state is coupled to two excited.

In this paper, we wish to study the squeezing, entanglement, and statistical properties of two-mode light produced by V-type three-level laser coupled to squeezed vacuum reservoir by using the expectation value of the cavity mode operators at steady state.

\section{Radiation of V-type Three Level Laser}

\subsection{Quantum Hamiltonian}

The interaction of one electron-atom having position $(\hat{r})$ and charge (e) with a radiation represented by vector potential $(\hat{A})$ is described by the Hamiltonian [15]

$$
\hat{\mathrm{H}}_{I}=-e \hat{\mathrm{r}} \frac{d}{d t} \hat{\mathrm{A}}
$$

In dipole approximation, the vector potential does not depend on the position coordinates

$$
\frac{d}{d t} \hat{\mathrm{A}}=\frac{\partial}{\partial t} \hat{\mathrm{A}}=-\hat{\mathrm{E}}
$$


The vector potentials for two-mode light is given by

$$
\begin{aligned}
\hat{\mathrm{A}}=A\left(\hat{\mathrm{a}} e^{-i \omega_{a} t}+\hat{\mathrm{a}}^{+} e^{i \omega_{a} t}\right) & +B\left(\hat{b} e^{-i \omega_{b} t}+\hat{b}^{+} e^{i \omega_{b} t}\right) \hat{u}(3) \\
\hat{\mathrm{E}} & =i\left(A \omega_{a}\left(\hat{\mathrm{a}} e^{-i \omega_{a} t}-\hat{\mathrm{a}}^{+} e^{i \omega_{a} t}\right)+B \omega_{b}\left(\hat{b} e^{-i \omega_{b} t}-\hat{b}^{+} e^{i \omega_{b} t}\right) \hat{u}\right.
\end{aligned}
$$

The Hamiltonian of the atom can be expressed as

$$
H_{A}=\hat{\sigma}_{a a} E_{a}+\hat{\sigma}_{b b} E_{b}+\hat{\sigma}_{c c} E_{c}
$$

The position operator for atomic electron defined as

$$
r(t)=r_{a c} e^{-i \omega_{a c} t}+r_{c a} e^{-i \omega_{a c} t}+r_{b c} e^{-i \omega_{b c} t}+r_{c b} e^{-i \omega_{b c} t}
$$

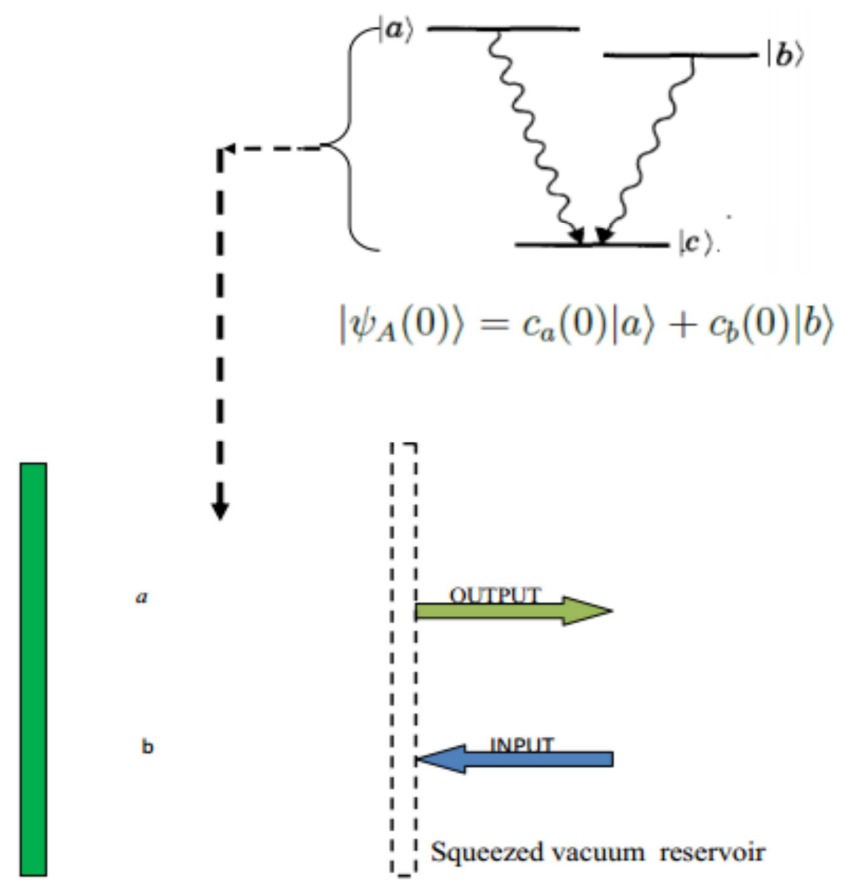

Figure 1. Schematic diagram of V-type three-level laser coupled to squeezed vacuum reservoir.

The Hamiltonian of V-type three-level laser expressed as

$$
\widehat{H}_{I}=\operatorname{ig}_{a c}\left(\hat{\sigma}_{a c} \hat{a} e^{i\left(\omega_{a c-w_{a}}\right) t}-\hat{\sigma}_{c a} \hat{\mathrm{a}}^{+} e^{-i\left(\omega_{a c}-w_{a}\right) t}\right)+\operatorname{ig}_{\mathrm{bc}}\left(\hat{\sigma}_{b c} \hat{b} e^{i\left(\omega_{b c-w_{b}}\right) t}+\hat{\sigma}_{c b} \hat{b}^{+} e^{-i\left(\omega_{b c}-w_{b}\right) t}\right)
$$

Where, $g f h_{a c}=A \omega_{a} e r_{a c} . \hat{u}$ and $g_{b c}=B \omega_{b} e r_{b c} . \hat{u}$ are known as the coupling constants.

\subsection{Master Equation}

The interaction of V-type three-level atom with cavity modes at resonance can be described by Hamiltonian

$$
H_{I}=\operatorname{ig}_{\mathrm{ac}}\left(\hat{\sigma}_{a c} \hat{\mathrm{a}} e^{i\left(\omega_{a c}-w_{a}\right) t}-\hat{\sigma}_{c a} \hat{\mathrm{a}}^{+} e^{-i\left(\omega_{a c}-w_{a}\right) t}+\operatorname{ig}_{\mathrm{bc}}\left(\hat{\sigma}_{b c} \hat{b} e^{i\left(\omega_{b c-w_{b}}\right) t}-\hat{\sigma}_{c b} \hat{b}^{+} e^{-i\left(\omega_{b c}-w_{b}\right) t}\right)\right.
$$

We take the initial state of V-type three-level atoms to be

$$
\psi_{A}(0)=c a(0)|a>+c b(0)| b>
$$

The density operator for a single atom is

$$
\rho_{A}^{0}=\rho_{a a}^{0}|\mathrm{a}><\mathrm{a}|+\rho_{a b}^{0}|\mathrm{a}><\mathrm{b}|+\rho_{b a}^{0}|b><a|+\rho_{b b}^{0}|b><b|
$$

Where, $\rho_{a a}^{0}=c a^{2}$ and $\rho_{b b}^{0}=c b^{2}$ are the probability for the atom to be in state $a_{i}$ and $b_{i}$ at the initial time, respectively and $\rho_{a b}(0)^{2}=c_{a} c_{b}$ represents the atomic coherence at the initial time with the relation,

$$
\rho_{a b}(0)^{2}=\rho_{a a}(0) \rho_{b b}(0)
$$

The master equation for V-type three-level laser coupled to a two-mode squeeze vacuum reservoir can be defined as 
$\frac{d}{d t} \rho=\frac{1}{2}\left(k N+A \rho_{a a}^{0}\right)\left(2 \hat{a}^{+} \rho \mathrm{a}-\hat{a} \hat{a}^{+} \rho-\rho \hat{a} \hat{a}^{+}\right)+\frac{1}{2}\left(k N+A \rho_{b b}^{0}\right)\left(2 \hat{b}^{+} \rho \hat{b}-2 \hat{b} \hat{b}^{+} \rho-\right.$
$\left.2 \rho \hat{b} \hat{b}^{+}\right)+\frac{1}{2}\left(A \rho_{a b}^{0}\right)\left(2 \hat{a}^{+} \rho \hat{b}-2 \hat{b}^{+} \rho \hat{a}^{+}-2 \rho \hat{b} \hat{a}^{+}\right)+\frac{1}{2} A \rho_{b a}\left(2 \hat{b}^{+} \rho \mathrm{a}-2 \hat{a} \hat{b}^{+} \rho-2 \rho \hat{a} \hat{b}^{+}\right)+\frac{1}{2} k(N+1)\left(2 \mathrm{a} \rho \hat{a}^{+}-2 \hat{a}^{+} \hat{a} \rho-\right.$ $\left.2 \rho \hat{a}^{+} \hat{a}\right)+\frac{1}{2} k(N+1)\left(2 \widehat{b} \rho \hat{b}^{+}-2 \hat{b}^{+} \hat{b} \rho-2 \rho \hat{b}^{+} \hat{b}\right)+1 / 2 k M\left(2 \hat{a}^{+} \hat{b}^{+} \rho-\hat{a}^{+} \hat{b}^{+} \rho-\rho \hat{a}^{+} \hat{b}^{+}+2 \hat{a} \hat{b} \rho-\hat{a} \hat{b} \rho-\rho \mathbf{a} \hat{b}\right)+\frac{1}{2} k M\left(2 \hat{b}^{+} \hat{a}^{+} \rho-\hat{a}^{+} \hat{b}^{+} \rho-\right.$ $\left.\rho \hat{a}^{+} \hat{b}^{+}+2 \widehat{b} \hat{a} \rho-\hat{a} \hat{b} \rho-\rho \hat{a} \widehat{b}\right)$

Where $k$ is the cavity decay constant, A is the linear gain coefficient, $\mathrm{r}$ is being the squeeze parameter, $\mathrm{N}=\sinh ^{2}(r)$ and $M=\sinh (r) \cosh (r)$ are reservoir parameters.

\subsection{Expectation Values}

The solution of the expectation value of the cavity mode operators using Eq. (12) and trace operation

$$
\begin{gathered}
\frac{d}{d t}<\mathrm{a}>-\frac{1}{2} \mu_{a}<\hat{\mathrm{a}}>+\frac{1}{2} v<\hat{b}>, \\
\frac{d}{d t}<\hat{b}>=-\frac{1}{2} \mu_{b}<\hat{b}>+\frac{1}{2} v<\hat{\mathrm{a}}>, \\
\frac{d}{d t}<\hat{\mathrm{a}}^{2}>=-\mu_{a}<\hat{\mathrm{a}}^{2}>+v<\hat{\mathrm{a}} \hat{b}>, \\
\frac{d}{d t}<\hat{b}^{2}>=-\mu_{b}<\hat{b}^{2}>+v<\mathrm{a} \hat{b}>, \\
\frac{d}{d t}<\hat{\mathrm{a}}^{+} \hat{\mathrm{a}}>=-\mu_{a}<\hat{\mathrm{a}}^{+} \hat{\mathrm{a}}>+\frac{1}{2} v\left(<\hat{\mathrm{a}}^{+} \hat{b}>+<\hat{b}^{+} \hat{\mathrm{a}}>\right)+k N, \\
\frac{d}{d t}<\hat{b}^{+} \hat{b}>=-\mu_{b}<\hat{b}^{+} \hat{b}>+\frac{1}{2} v\left(<\hat{\mathrm{a}}^{+} \hat{b}>+<\hat{b}^{+} \hat{\mathrm{a}}>\right)+k N, \\
\frac{d}{d t}<\mathrm{a} \hat{b}>=-\frac{1}{2}\left(\mu_{a}+\mu_{b}\right)<\mathrm{a} \hat{b}>+\frac{1}{2}<v>\left(<\hat{\mathrm{a}}^{2}>+<\hat{b}^{2}>\right)-k M, \\
\frac{d}{d t}<\hat{\mathrm{a}}^{+} \hat{b}>=-\frac{1}{2}\left(\mu_{a}+\mu_{b}\right)<\hat{\mathrm{a}}^{+} \hat{b}>+\frac{1}{2}<v>\left(<\hat{\mathrm{a}}^{+} \hat{\mathrm{a}}>+<\hat{b}^{+} \hat{b}>\right),
\end{gathered}
$$

Where $\mu_{a}=k-A \rho_{a a}^{0}, \mu_{b}=k-A \rho_{a a}^{0}$ and $v=A \rho_{a b}^{0}=$ $A \rho_{b a}^{0}$.

The steady state solution of the expectation value of the system have a solution by considering the case for $\mu_{a} \mu_{b}>$ $v^{2}$, and the solution

$$
\begin{gathered}
<\hat{\mathrm{a}}>=<\hat{b}>=0, \\
<\hat{\mathrm{a}}^{2}>=-\frac{2 v \mu_{b} k M}{\left(\mu_{a}+\mu_{b}\right)\left(\mu_{a} \mu_{b}-v^{2}\right)^{\prime}} \\
<\hat{b}^{2}>=-\frac{2 v \mu_{a} k M}{\left(\mu_{a}+\mu_{b}\right)\left(\mu_{a} \mu_{b}-v^{2}\right)^{\prime}} \\
<\hat{\mathrm{a}}^{+} \hat{\mathrm{a}}>=\frac{\mu_{b} k N\left(\mu_{a}+\mu_{b}\right)}{\left(\mu_{a}+\mu_{b}\right)\left(\mu_{a} \mu_{b}-v^{2}\right)^{\prime}} \\
<\hat{b}^{+} \hat{b}>=\frac{\mu_{a} k N\left(\mu_{a}+\mu_{b}\right)}{\left(\mu_{a}+\mu_{b}\right)\left(\mu_{a} \mu_{b}-v^{2}\right)^{\prime}} \\
<\hat{\mathrm{a}}^{+} \hat{b}>=\frac{v k N\left(\mu_{a}+\mu_{b}\right)}{\left(\mu_{a}+\mu_{b}\right)\left(\mu_{a} \mu_{b}-v^{2}\right)^{\prime}}
\end{gathered}
$$

$$
<\hat{\mathrm{a}} \hat{b}>=-\frac{2 \mu_{a} \mu_{b} k M}{\left(\mu_{a}+\mu_{b}\right)\left(\mu_{a} \mu_{b}-v^{2}\right)} .
$$

\section{Quadrature Squeezing}

The quadrature squeezing property of two-mode light is described by two Hermitian quadrature operators defined by

$$
\hat{c}_{ \pm}=\sqrt{ \pm 1}\left(\widehat{c}^{+} \pm \hat{c}\right),
$$

Where,

$$
\hat{c}=\frac{1}{\sqrt{2}}(\hat{\mathrm{a}}+\hat{b}) \text {. }
$$

The variance of the quadratures defined by

$$
\Delta c_{ \pm}^{2}=<\hat{c}_{ \pm}, \hat{c}_{ \pm}>
$$

we can be expressed as

$$
\begin{gathered}
\Delta \hat{c}_{ \pm}=1+\frac{1}{2}\left(2<\hat{\mathrm{a}}^{+}, \hat{\mathrm{a}}>+2<\hat{b}^{+}, \hat{b}>+2<\hat{\mathrm{a}}^{+}, \hat{b}>+2<\hat{b}^{+}, \hat{\mathrm{a}}\right) \pm<\hat{\mathrm{a}}^{+}, \hat{\mathrm{a}}^{+}> \pm<\mathrm{a}, \hat{\mathrm{a}}> \pm<\hat{b}^{+}, \hat{b}^{+}> \pm<\widehat{b}, \hat{b}> \pm 2< \\
\left.\hat{\mathrm{a}}^{+}, \hat{b}^{+}> \pm 2<\hat{\mathrm{a}}, \hat{b}>\right)
\end{gathered}
$$

Using Eqs. (21) - (27), the quadrature variance can be defined as

$$
\Delta \widehat{c}_{ \pm}^{2}=\frac{(2 k-A)\left(k-A+\left(2 k-A+A \sqrt{1-\eta^{2}}\right)(N \mp M) \pm M A^{2} \eta^{2}\right)}{(2 k-A)(k-A)} .
$$

Where, $\rho_{b b}^{0}-\rho_{a a}^{0}=\eta$ and $\rho_{a b}^{0}=\frac{1}{2} \sqrt{1-\eta^{2}}$ 


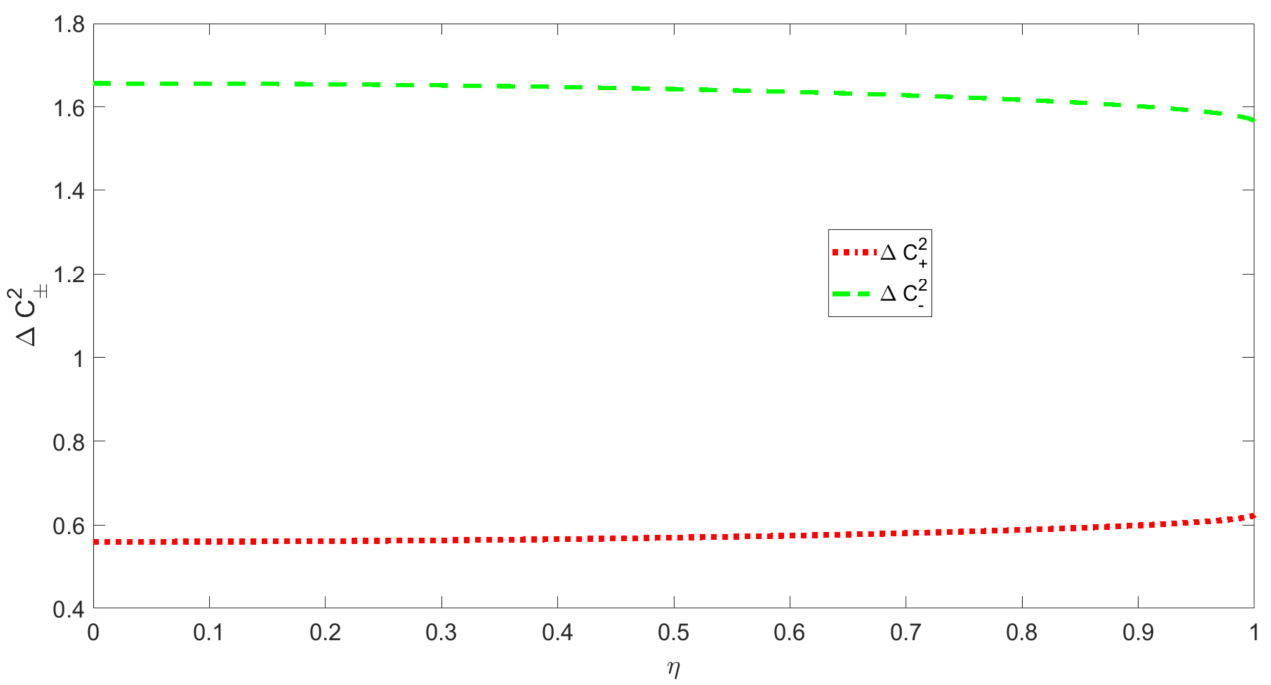

Figure 2. Represent the plots of the quadrature variance [Eq. (32)] versus $\eta$ for $k=0.8, r=0.2$ and $A=0.5$.

The figure shows that the system under consideration can produce squeezed light and the squeezing occurs in the plus quadrature.

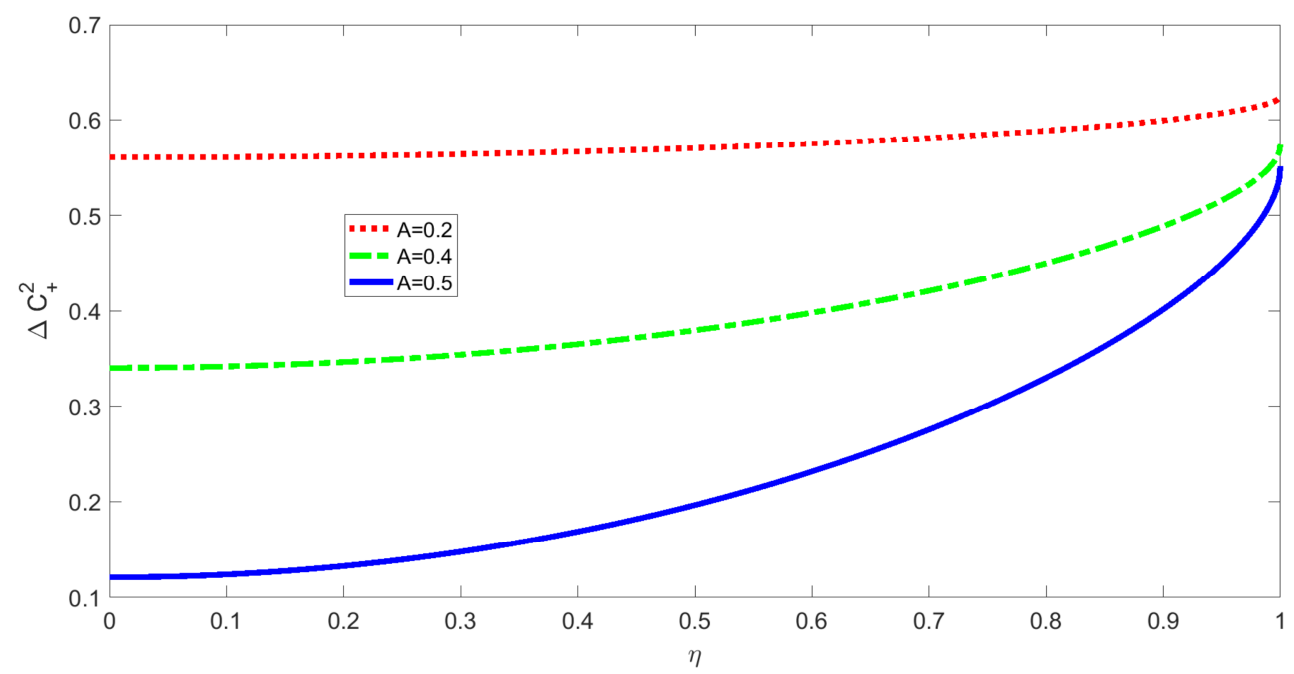

Figure 3. Represent the plots of the quadrature squeezing [Eq. (35)] versus $\eta$ for $k=0.8, r=0.2$ and for different value of $A$.

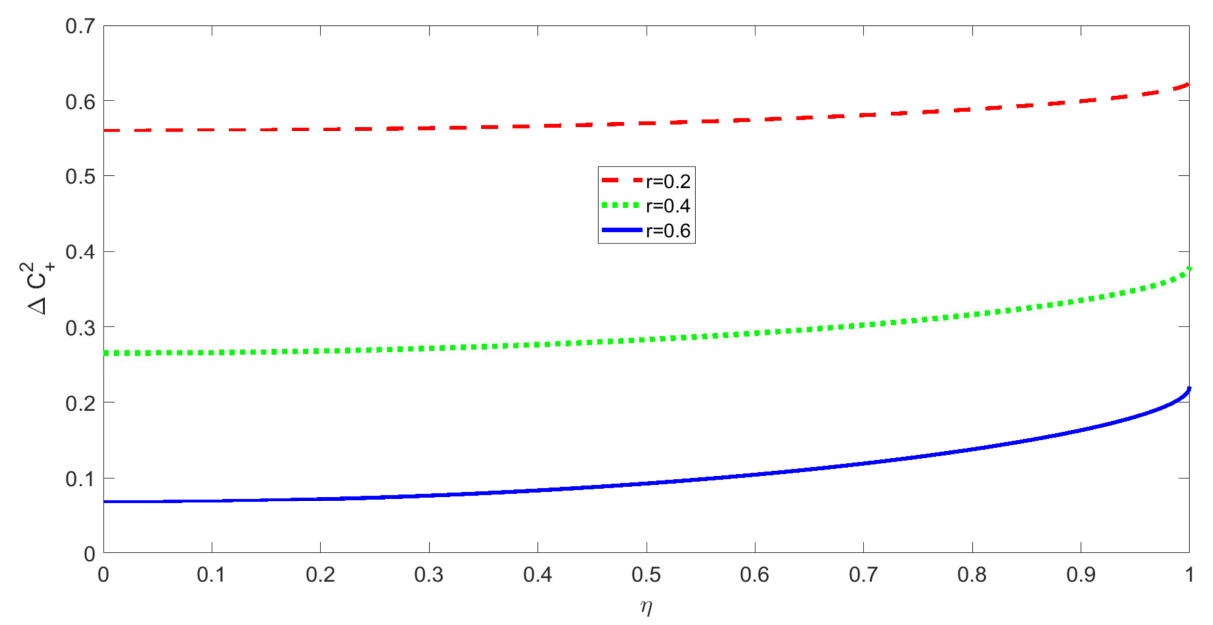

Figure 4. Represent the plots of the plus quadrature variance [Eq. (32)] versus $\eta$ for $k=0.8, A=0.5$ and for different squeezing parameter. 
Figure 3 and Figure 4 shows that the quadrature variance increase with $\eta$, and decreases as the linear gain coefficient and squeezing parameter.

The quadrature squeezing of a two-mode cavity light relative to the quadrature variance of the two-mode coherent (vacuum) light can be defined as

$$
S=\frac{\Delta \widehat{c}_{ \pm v}^{2}-\Delta \hat{c}_{+}}{\left(\Delta \hat{c}_{ \pm}\right)_{v}^{2}}
$$

where

$$
\Delta \hat{c}_{ \pm v}^{2}=1
$$

is the quadrature variance for coherent (vacuum) state.

With the aid of Eqs. (32), (33), and (34), the quadrature squeezing relative to coherent (vacuum) state

$$
S=\frac{(2 k-A)\left(2 k-A+A \sqrt{1-\eta^{2}}\right)(N-M)+M A^{2} \eta^{2}}{(2 k-A)(k-A)} .
$$

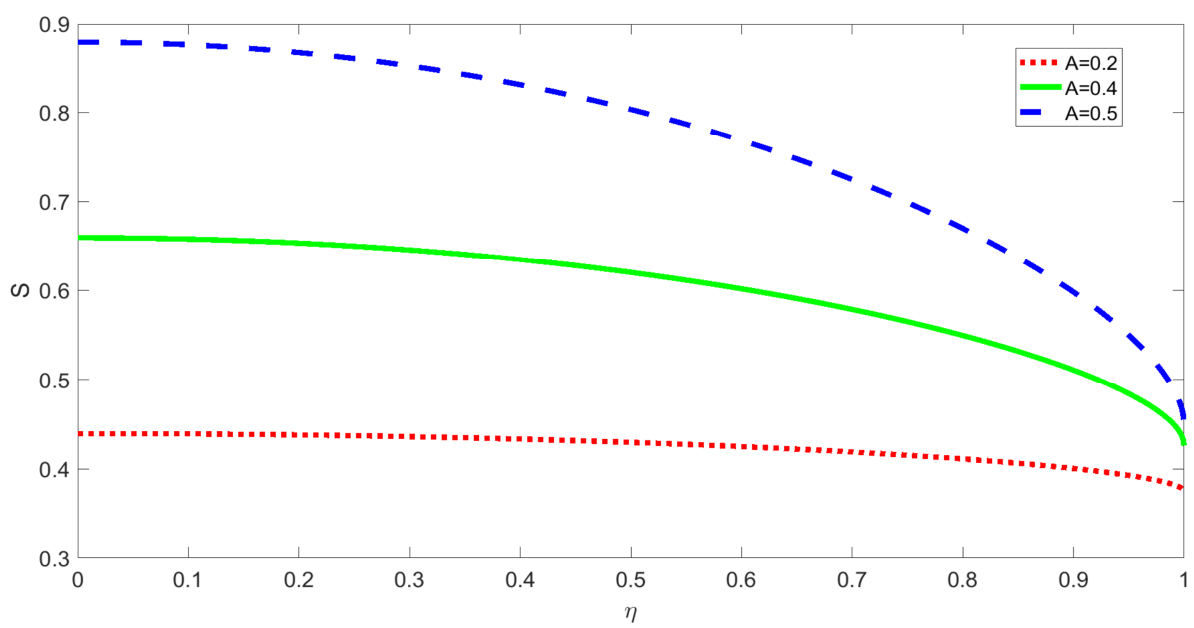

Figure 5. Represent the plots of the quadrature squeezing [Eq. (35)] versus for $k=0.8, r=0.2$ and for different value of $A$.

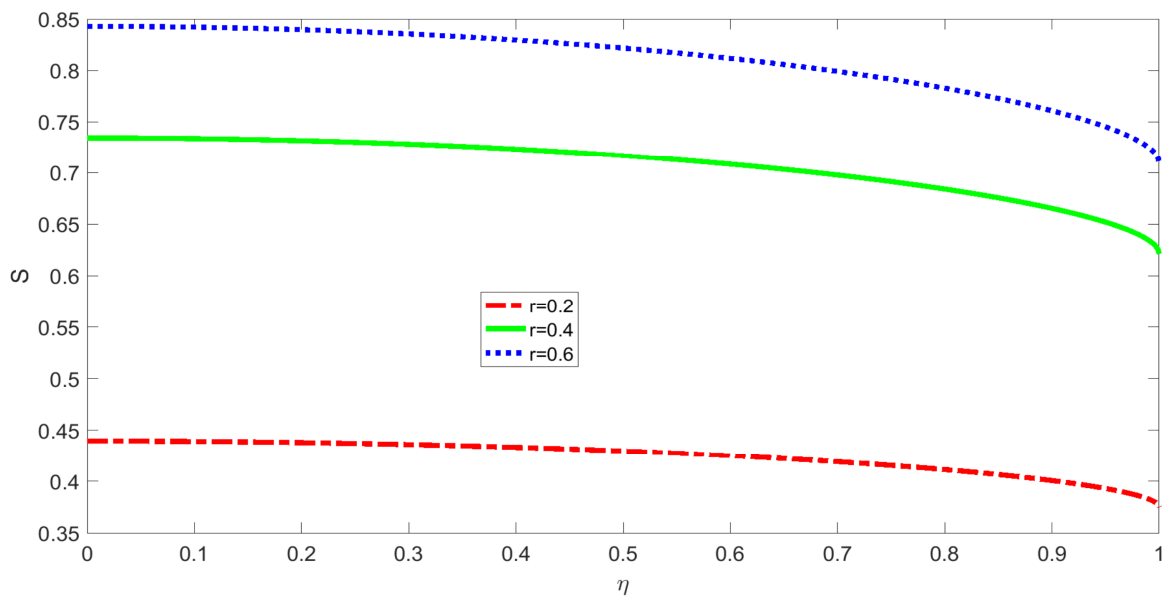

Figure 6. Represent the plots of the quadrature squeezing [Eq.(35)] versus $\eta$ for $k=0.8, A=0.2$ for different squeezing parameter.

Figures 5 and 6 shows that the degree of squeezing increases with linear gain coefficient and squeezing parameter.

\section{Entanglement}

The EPR like operators can be expressed in the form

$$
\begin{aligned}
& \hat{\mathrm{U}}=\hat{\mathrm{a}}_{+}-\hat{b}_{+} \\
& \hat{v}=\hat{\mathrm{a}}_{-}-\widehat{b}_{-}
\end{aligned}
$$

The variance of EPR-type variables can be defined as

$$
\begin{aligned}
& \Delta \hat{u}^{2}=\langle\hat{u}, \hat{u}> \\
& \Delta \hat{v}^{2}=\langle\hat{v}, \hat{v}>
\end{aligned}
$$

Adding Eqs. (38) and (39), we have

$$
\Delta \hat{u}^{2}+\Delta \hat{v}^{2}=2+\frac{2(2 k-A)^{2}(N+M)-2 A M \eta^{2}}{(2 k-A)(k-A)} .
$$

Eq. (40) represents the sum of the variances of EPR-like variables for a two-mode light produced by V-type threelevel Laser with squeezed vacuum reservoirs. We easily see from the equation that $\Delta \hat{u}^{2}+\Delta \hat{v}^{2}>2$. This result shows that the cavity modes produced by the system under consideration 
do not exhibited entanglement.

\section{Statistical Properties}

To study the statistical properties of a two-mode light produced by V-type three-level laser coupled to squeezed vacuum reservoir.

\subsection{The Mean Photon Number}

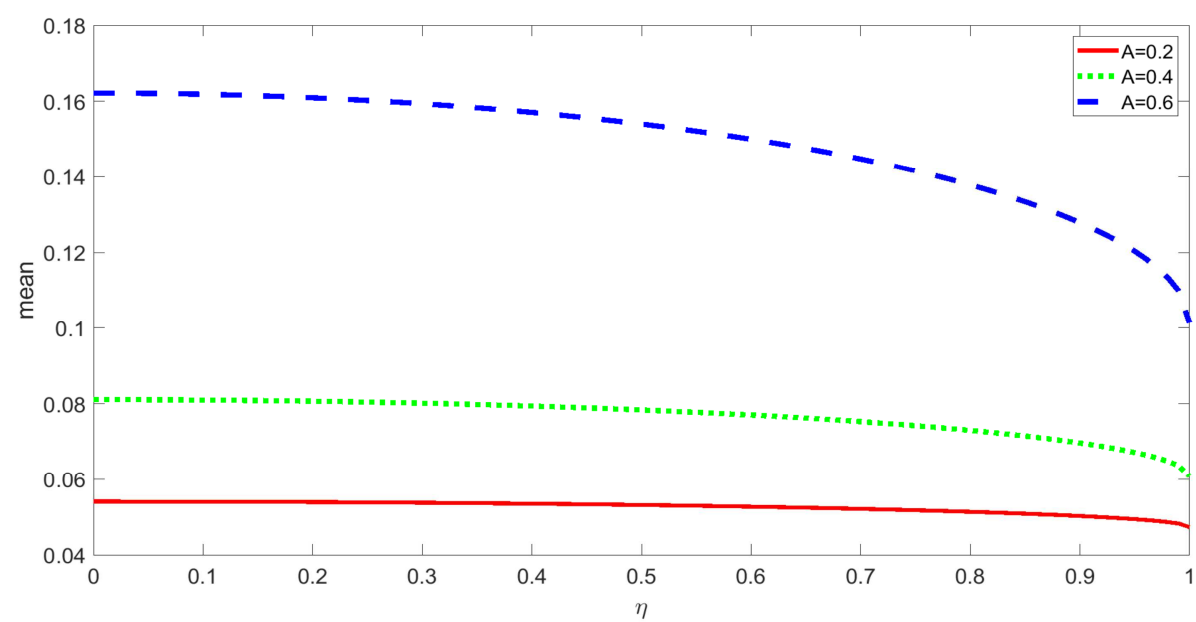

Figure 7. Represent the plots of the mean photon number [Eq. (40)] versus $\eta$ for $r=0.2, \kappa=0.8$, and different values of $A$.

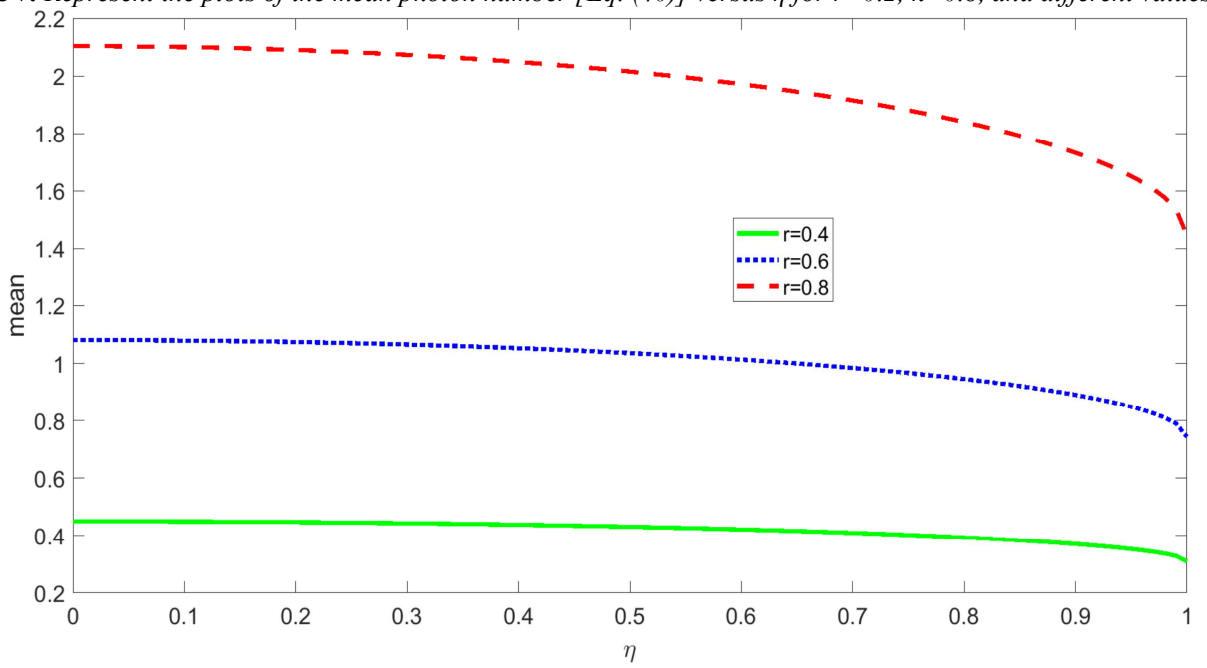

Figure 8. Represent the Plots of the mean photon number [Eq. (40)] versus $\boldsymbol{\eta}$ for $k=0.8, A=0.2$ and different values of $r$.

Figure 7. shows that the mean photon number decreases as $\eta$ but increases with linear gain coefficient.

Figure 8 . shows that the mean photon number increase with squeezing parameter.

\subsection{The Variance of Photon Number}

The variance of the photon number can be defined by
The mean photon number for a two-mode light can be defined as

$$
\bar{n}=\left\langle\hat{c}^{+} \hat{c}>,\right.
$$

The mean photon number at steady state to be

$$
\bar{n}=\frac{N\left(2 k-A+A \sqrt{1-\eta^{2}}\right.}{2(k-A)} .
$$




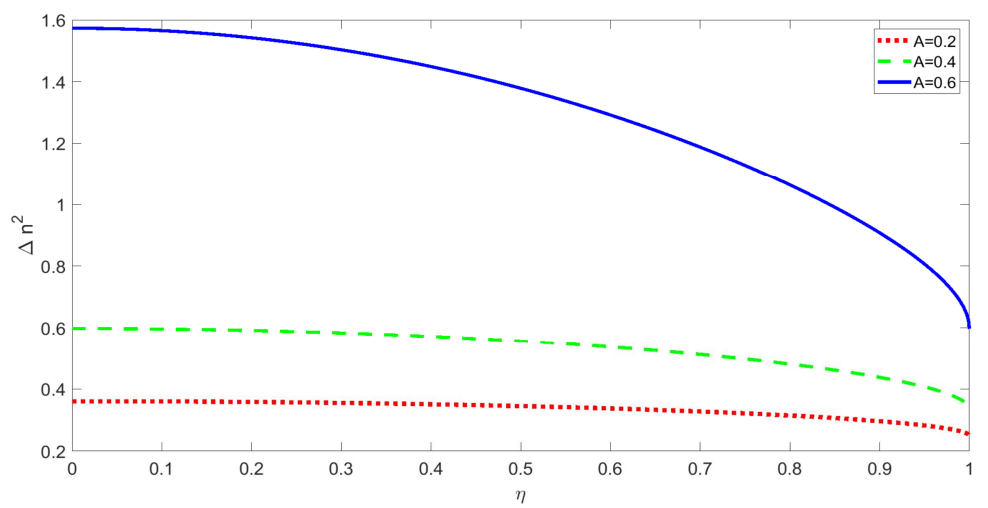

Figure 9. Represent the plots the variance of photon number [Eq. (45)] versus $\boldsymbol{\eta}$ for $k=0.8, r=0.2$ and for different values of $A$.

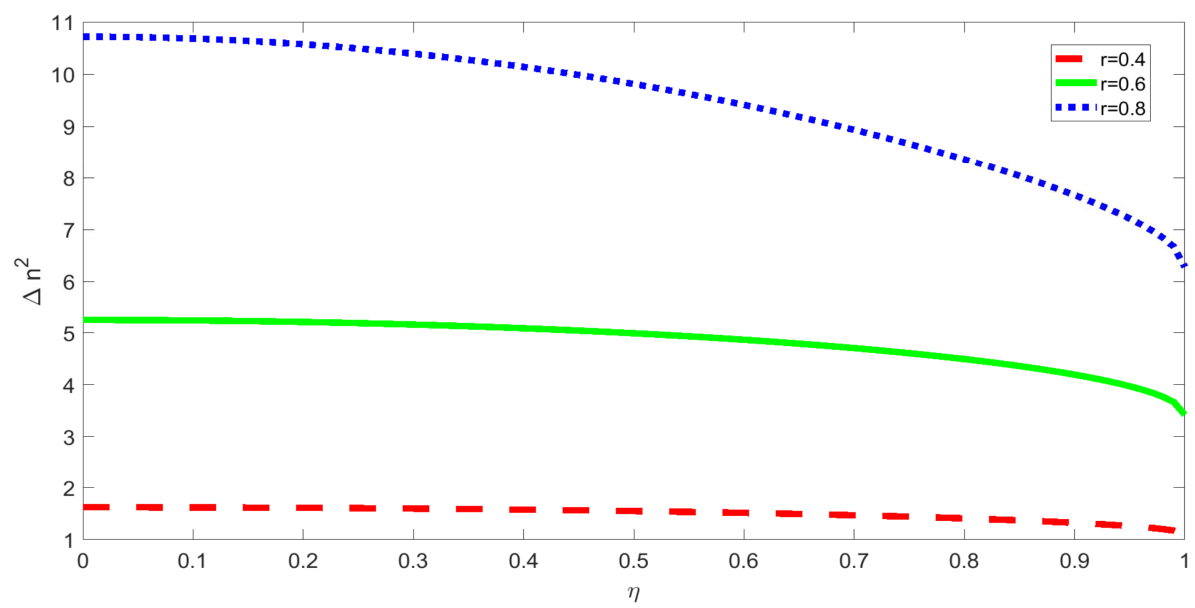

Figure 10. Represent the plots the variance of photon number [Eq. (45)] versus $\boldsymbol{\eta}$ for $k=0.8, r=0.2$ and for different values of $r$.

Figures 9 and 10. shows that variance of photon number increase with linear gain coefficient and squeeze parameter.

\subsection{Intensity Difference Fluctuations}

The variance of the intensity difference can be defined by

$$
\Delta I_{d}^{2}=<\hat{\mathrm{a}}^{+} \hat{\mathrm{a}}>^{2}+<\hat{b}^{+} \hat{b}>^{2}+<\hat{\mathrm{a}}^{+} \hat{\mathrm{a}}>+<\hat{b}^{+} \hat{b}>+\left|<\hat{\mathrm{a}}^{2}>\right|^{2}+\left|<\hat{b}^{2}>\right|^{2}-2|<\hat{\mathrm{a}} \hat{b}>|^{2}-2\left|<\hat{\mathrm{a}}^{+} \hat{b}>\right|^{2},
$$

Applying Eqs. (22) - (27) into (48), we have

$$
\Delta I_{d}^{2}=\frac{M^{2} \eta^{2}\left(10 A^{2}-24 \kappa^{2} A+8 \kappa A-8 A^{4} \eta^{2}-16 \kappa A^{3}\right)}{8(2 \kappa-A)^{2}(\kappa-A)^{2}}+\frac{M^{2}\left(A^{2}\left(40 \kappa^{2}-24 \kappa A+33 A^{2}\right)+16 \kappa^{3}(\kappa-2 A)\right)}{8(2 \kappa-A)^{2}(\kappa-A)^{2}}+\frac{N^{2}\left(2 \kappa(1-A)+A^{2}\left(\frac{3}{4}+5 \eta^{2}\right)\right)}{(\kappa-A)^{2}}+\frac{\kappa N}{2 \kappa-A}
$$

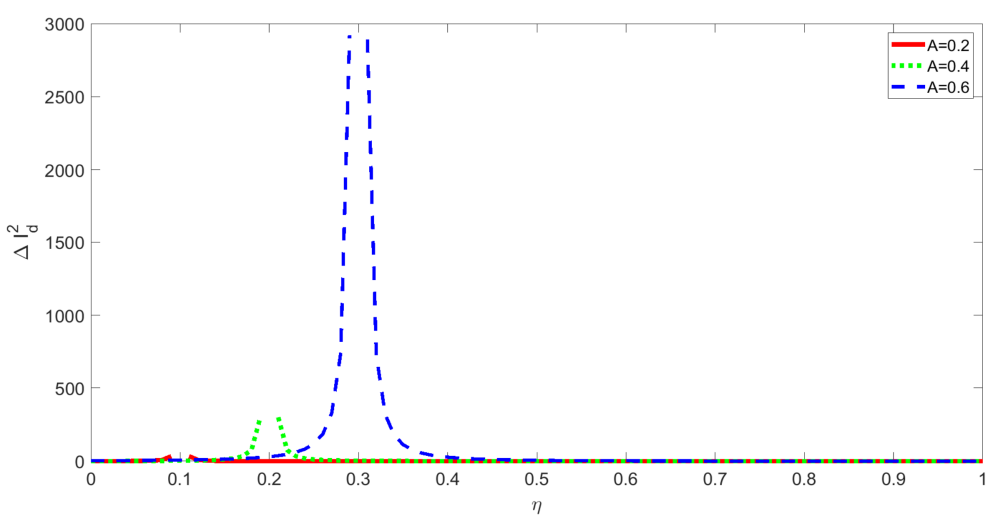

Figure 11. Represent plots the variance of intensity difference [Eq.(49)] versus $\boldsymbol{\eta}$ for the value of $k=0.8, r=0.2$ and different values of linear gain coefficient. 


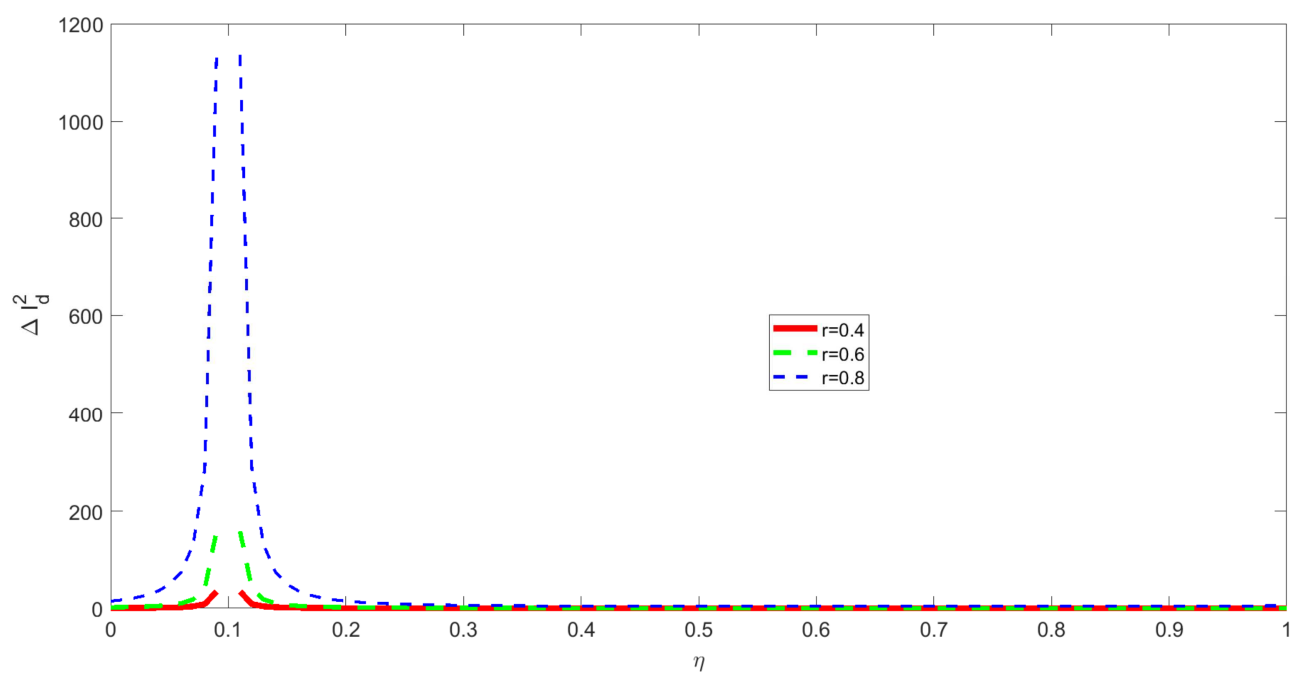

Figure 12. Represent the plots of the variance of intensity difference versus $\boldsymbol{\eta}$ for different values squeezing parameter with $k=0.8$ and $r=0.2$

Figure 11 and Figure 12 indicates that the point $\boldsymbol{\eta}$, at which the maximum intensity difference fluctuation occurred depends on linear gain coefficient but not on the squeezing parameter.

\subsection{Second Order Correlation Function}

The second-order correlation function for the two-mode radiation at equal time is given

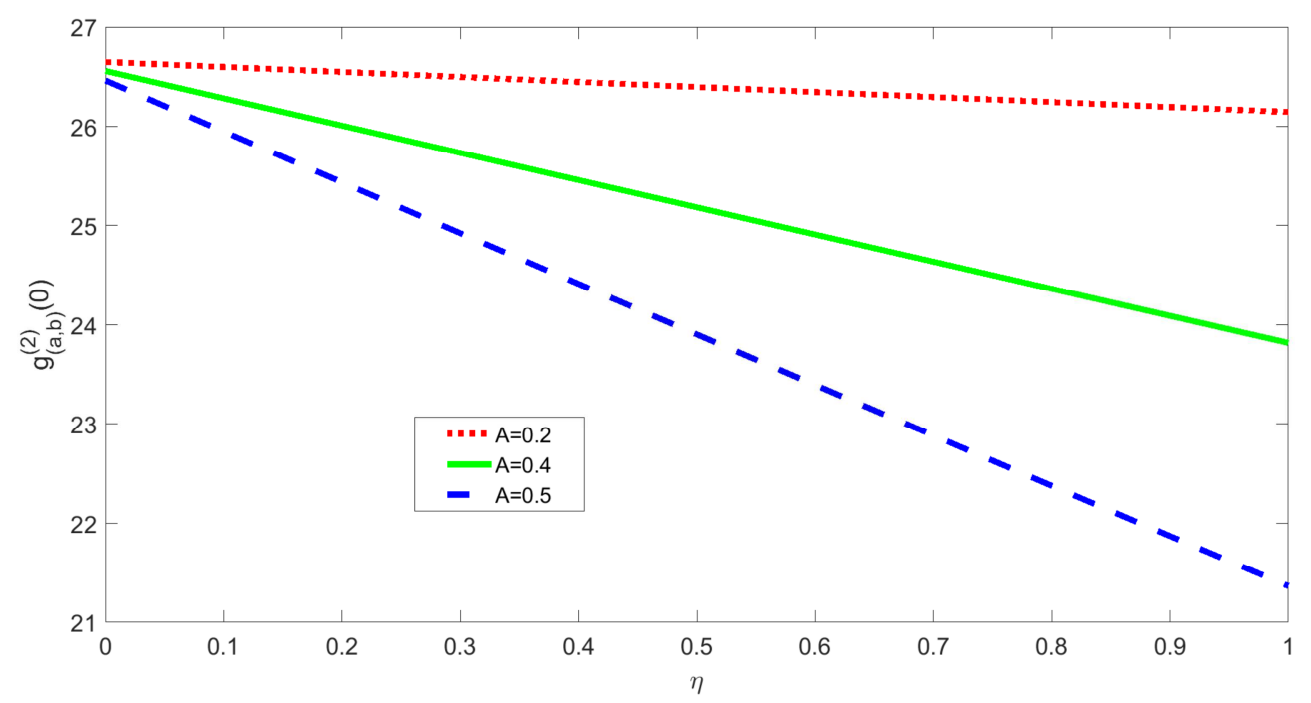

Figure 13. Represent the plots of second-order correlation function [Eq. (50)] versus $\boldsymbol{\eta}$ for $k=0.8, r=0.2$ and for different values of $A$.

$$
g_{(a, b)}^{(2)}(0)=\frac{<\hat{a}^{+} \hat{a} \widehat{b}^{+} \widehat{b}>}{<\hat{a}^{+} \hat{a}><\widehat{b}^{+} \hat{b}>}
$$

Since â and $\hat{b}$ are Gaussian variable with vanishing means, this equation can be written as

$$
g_{(a, b)}^{(2)}(0)=1+\frac{<\hat{a} \hat{b}>^{2}+<\hat{a}^{+} \widehat{b}>^{2}}{<\hat{a}^{+} \hat{a}><\widehat{b}^{+} \hat{b}>}
$$

On account of Eq. (24) -(27), we find Eq. (51)

$$
g_{(a, b)}^{(2)}(0)=1-\frac{A^{2}(1-\eta)}{\left.(2 \kappa-A)^{2}-A^{2} \eta\right)}+\frac{M^{2}\left((2 \kappa-A)^{2}-A^{2} \eta^{2}\right)^{2}}{N^{2}(2 \kappa-A)^{2}\left((2 \kappa-A)^{2}-A^{2} \eta\right)}
$$

Eq. (52) represents the second-order correlation function of the two-modes light produced by V-type three-level laser coupled to squeezed vacuum reservoir. 


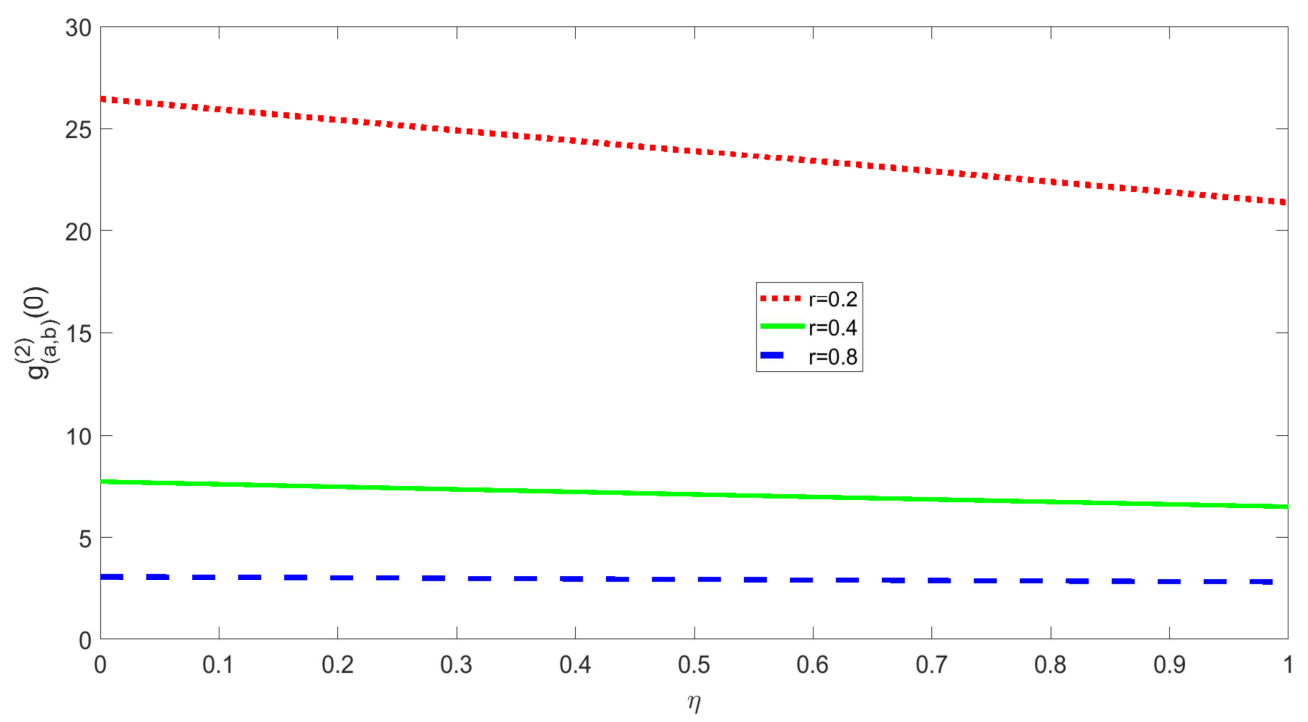

Figure 14. Represent the plots of second order correlation function [Eq. (50)] versus $\boldsymbol{\eta}$ for $k=0.8, A=0.2$ and different values of squeezing parameter.

Figures 13 and 14 shows that the correlation function decrease as the linear gain coefficient and squeezing parameter.

\section{Conclusion}

It is found that the two-mode light produced by the system under consideration is in a squeezed state for the case the cavity decay constant is greater than linear gain coefficient and the squeezing occurs in the plus quadrature. It is observed that the degree of squeezing increase with the linear gain coefficient and squeezing parameter. The cavity modes do not exhibit entanglement properties. The mean and variance of the photon number calculation results indicate that the effect of linear gain coefficient and squeezing parameter are increase the mean and variance of the photon number and the cavity two-mode has superpoisonian photon statistics. The point, $\eta$, at which the maximum intensity difference fluctuation occurred depends on linear gain coefficient but not squeezing parameter. The result shows that the correlation of the two modes decreases as $\eta$, the linear gain coefficient and squeezing parameter increase.

\section{References}

[1] Haroches, S. Raimond, J. M exploring the quantum, oxford university press (2006).

[2] Nor Hazmin Bt. Sabri, Non-classical properties of light in atom cavity interaction scheme with time and intensity dependent coupling, kuala lumpur press (2013).

[3] Dawit Hiluf and Fesseha Kassahun, Degenerate three level laser coupled to squeezed vacuum reservoir, arXiv: 1709.10047v1, (October 17, 2018).

[4] Getachew A. Gebiru, Generation of bright squeezed light from $\mathrm{N}$ three-level atoms pumped by a coherent light, journal of quantum information science 6, 121-141 (June 30, 2016).
[5] M. R. Abbasiem, Thermal entanglement of a V-type threelevel atom and bimodal photons, Physica A 501 (2018) 69-77.

[6] M. A. Anton, F. Carreno and Oscar G. Calderon, squeezing spectra in a V-type three-level Atom interacting with a broadband squeezed field, Optics Communications 234, 281294 (February 2, 2004).

[7] Y. Fang and J. Jang, Quantum squeezing and entanglement from a two-mode phase sensitive amplifier via four-wave mixing in rubidium vapor New J. Phys, 17 (2015) 023027.

[8] Eyob Alebachew and Fesseha Kassahun, A degenerate three level laser with a parametric amplifier, arXiv: 10506178v3 (1, 2007).

[9] LI-Gao Xiang, PENG-Jin Sheng and WUS-Huang Guang Min, Population Inversion of a V-Type Three-Level Atom due to the effect of cavity induced quantum interference Vol, 18 , No 1 (2001).

[10] Habitamu Dagnaw and Sitotaw Eshete, Quantum Analysis of Degenerate Three-Level Laser, Advance in physics theory and applications 2225-0638 Vol, 72 (2018).

[11] M. O Scully and M. S. Zubarry, Quantum Optics (Cambridge university press, Cam-bridge, 1997).

[12] Sitotaw Eshete and Misrak Gatahun, Superposed two-mode squeezed laser light coupled to squeezed vacuum reservoir, Advance in physics theory and application 2225-0638 Vol, 71 (2018).

[13] Eyob Alebachew, Enhanced squeezing and entanglement in a non-degenerate three-level cascade laser with injected squeezed light, Optics Communications, Italy 280 (2007) 133141.

[14] Wen-Xing Yang, Ai-Xi Chen, Ting-Ting Zha, Yanfeng Bai, Ray-Kuang Lee, Interference-induced enhancement of field entanglement in a microwave-driven V-type single-atom laser, Central European Journal of Physics 12 (10), 2014, 737-743.

[15] Fesseha Kassahun, Fundamentals of Quantum Optics (Lulu Press Inc., North Car-olina, 2008). 